\title{
A Double Tension Band Technique for Patellar Fractures-A New Concept
}

\author{
Yogesh S. Salphale*, Nitin Kimmatkar, Hrishikesh R. Badve \\ Shushrusha Multispecialty Hospital, Chandrapur, India \\ Email: *yosa@aol.in
}

How to cite this paper: Salphale, Y.S., Kimmatkar, N. and Badve, H.R. (2020) A Double Tension Band Technique for Patellar Fractures-A New Concept. Surgical Science, 11, 127-132. https://doi.org/10.4236/ss.2020.116017

Received: May 20, 2020

Accepted: June 14, 2020

Published: June 17, 2020

Copyright $\odot 2020$ by author(s) and Scientific Research Publishing Inc. This work is licensed under the Creative Commons Attribution International License (CC BY 4.0).

http://creativecommons.org/licenses/by/4.0/

(c) (i) Open Access

\begin{abstract}
Amongst the many known techniques used for the management of fractures of the patella, the tension band wire fixation is a reliable treatment modality and has given consistently good results. In transverse patellar fractures, a combination of cerclage wiring and tension band fixation gives a good mechanical stability and an environment conducive to healing. The fracture described herein is classified as 45-C1 (based on Orthopaedic Trauma Association classification) of the right knee of a forty three years old male patient. It was fixed with three vertical $1.5 \mathrm{~mm} \mathrm{~K}$ wires and double cerclage wire looped proximally through the quadriceps tendon and distally through the patellar ligament in a double figure-of-eight configuration. The average follow-up period was 7 months. The Activity of Daily Living Scale (ADLS) of the Knee Outcome Survey was used to assess symptoms and functional capability of the knee. The fracture union was achieved at an average of 10 weeks. Full range of knee motion was achieved by end of the third postoperative month. No complications, such as infection and implant failure were noted. The technique aims to stress the advantage of giving a balanced fixation addressing the bony elements (the fractured ends) as well as the soft tissue (the extensor apparatus).
\end{abstract}

\section{Keywords}

Transverse Patella Fracture, Cerclage Wiring, Tension Band Wiring, Double Tension Band Wiring

\section{Introduction}

Patella fractures account for $1 \%$ of all skeletal fractures. Comminuted patellar fractures usually occur with direct trauma. About one third of patella fractures require surgery, which is indicated if there is damage to the extensor mechanism 
or in fractures associated with $2 \mathrm{~mm}$ step-off incongruity [1]. The objectives of surgical treatment include precise anatomic reduction of the articular surface by stable fixation, and restoration of the knee-extensor mechanism, thus allowing early mobilization [2]. Currently, several fixation methods of patellar fractures are in use, including tension band wiring, cerclage wiring, screw fixation, mini plate fixation and suture fixation and TBW with the use of cannulated screw [3].

\section{Surgical Technique of Modified Double Tension Band Wiring}

The surgery was performed with patient in the supine position with the injured knee extended under Spinal Anaesthesia. A longitudinal midline skin incision was made over the patella after exsanguinating the limb and placing the tourniquet. The X-ray was assessed (Figure 1). The proximal and distal fragments were reduced, restoring a smooth articular surface. They were held firmly with patella reduction clamps or towel clips. Three $2 \mathrm{~mm}$ Kirschner wires (K-wire) were made to pass from inferior to superior pole through each fragment keeping about $5 \mathrm{~mm}$ deep to the anterior surface of the patella and as parallel as possible (Figure 2(a)).

Then an 18-gauge stainless steel wire was passed transversely through the quadriceps tendon attachment, as close to the bone as possible, and deep to the $\mathrm{K}$-wires and then passed over the anterior surface of the patella. It was then routed

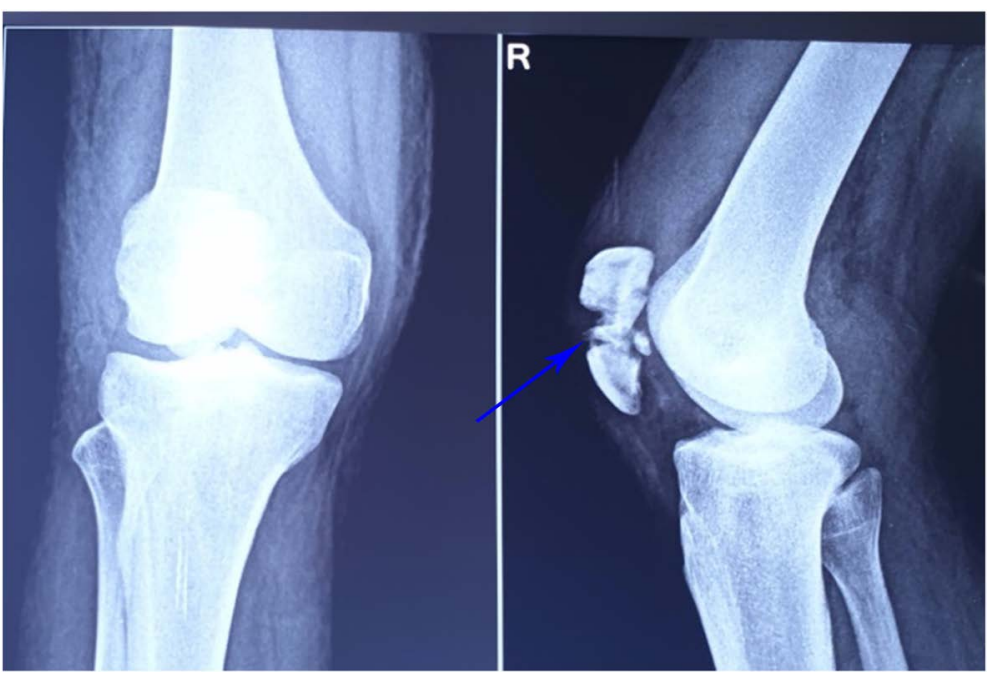

Figure 1. Pre op X-ray showing a fracture of patella with min Communition.

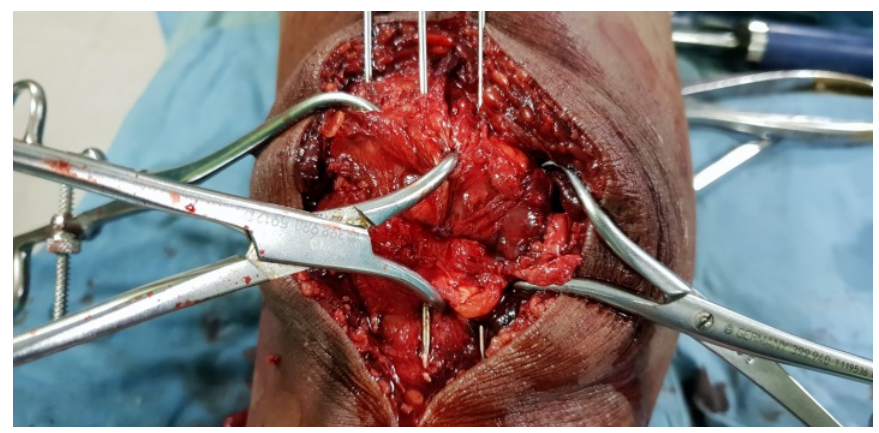

(a) 


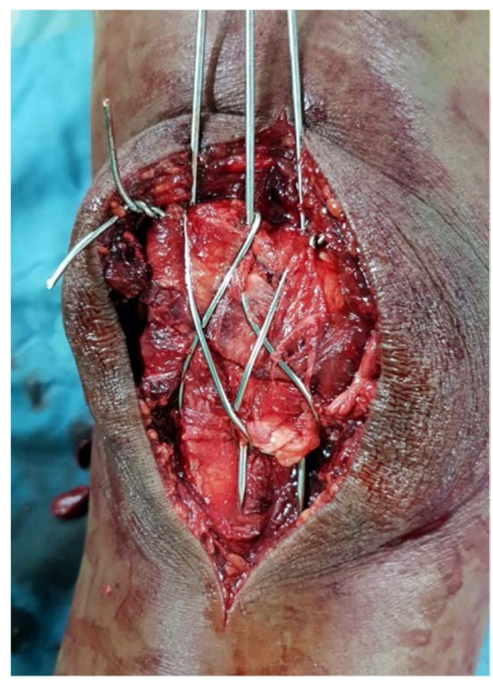

(b)

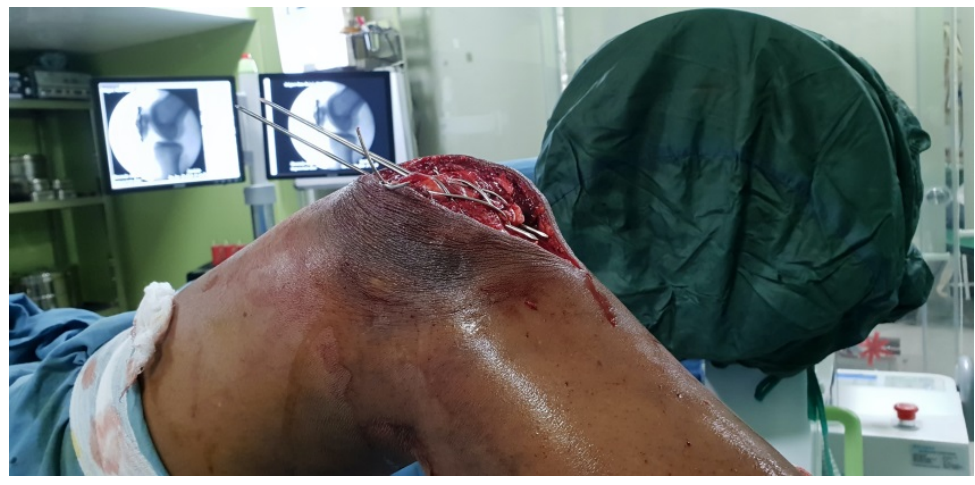

(c)

Figure 2. (a) Showing Intraoperative picture with fracture held reduced by clamps and three K wires; (b) Showing Intraoperative picture with fracture held reduced by clamps and three $\mathrm{K}$ wires with two wires in the double tension band assembly; (c) Showing Intraoperative picture with knee flexion and assessment of articular reduction.

transversely through the patellar tendon attachment on the inferior fragment and deep to the K-wires from lateral to medial side and then back over the anterior patellar surface. (Figure 2(b)). While tightening the second wire, congruity of the articular surface was checked by palpating the retro patellar surface. The repair of the retinaculum was done meticulously and the knee was flexed to around 80 degrees to ascertain the fixation. The $\mathrm{C}$ arm images were checked for the articular step. (Figure 2(c)). The closure of the wound was done. The upper ends of the three K-wires were embedded into the superior margin of the patella after bending it acutely and protruding ends of the K-wires were cut short inferiorly. Retinacular tears were repaired and the wound was closed over a suction drain. The post op X-ray was found satisfactory (Figure 3).

\section{Post operative protocol}

The operated knee was protected with a cylinder back slab. The sutures were removed after 12 days. The patient was encouraged with static Quadriceps exercises while in the slab. The slab was removed after 4 weeks. A course of physio- 
therapy that included knee bending, quadriceps and hamstring exercises were initiated. At the end of four months the patient had a good range of knee movements and a well healed scar (Figure 4(a) \& Figure 4(b)). The Activity of Daily Living Scale (ADLS) of the Knee Outcome Survey was used to assess symptoms

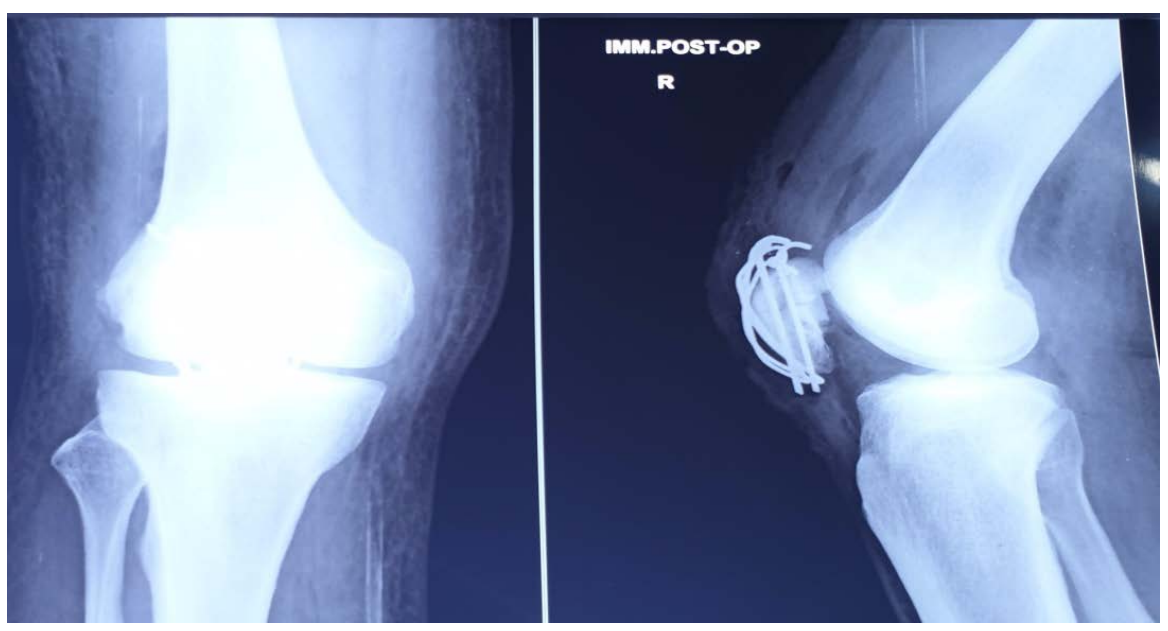

Figure 3. Showing X-ray picture with two wires in the double tension band assembly.

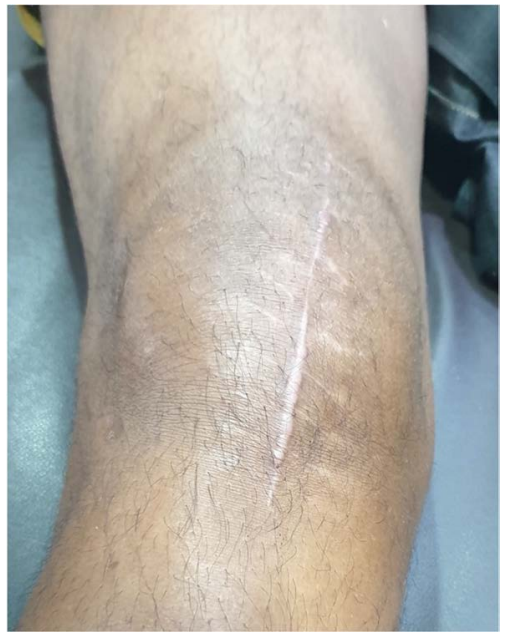

(a)

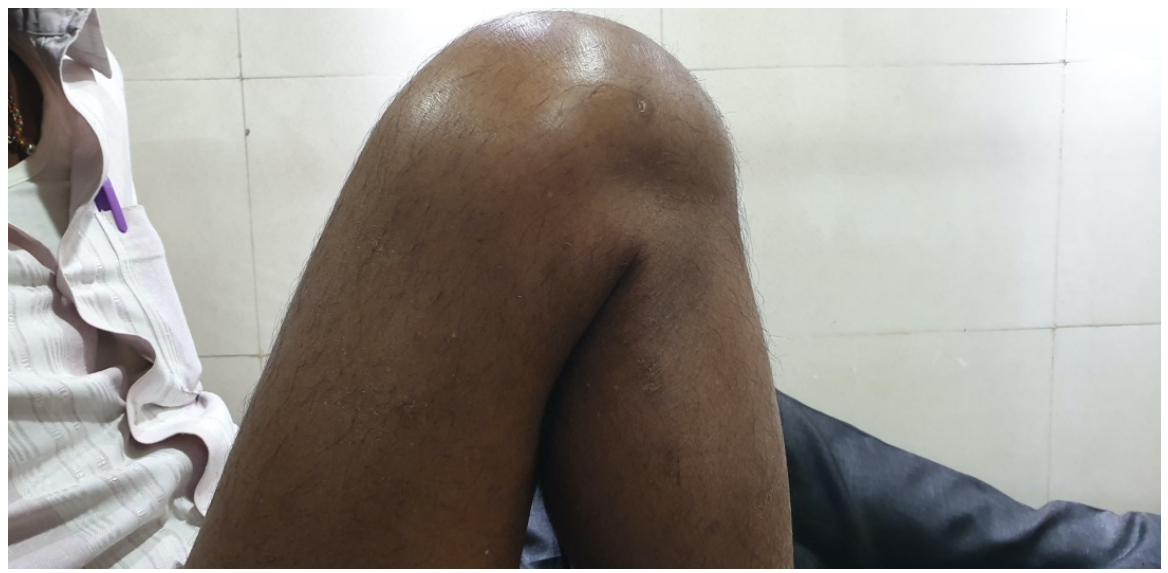

(b) 


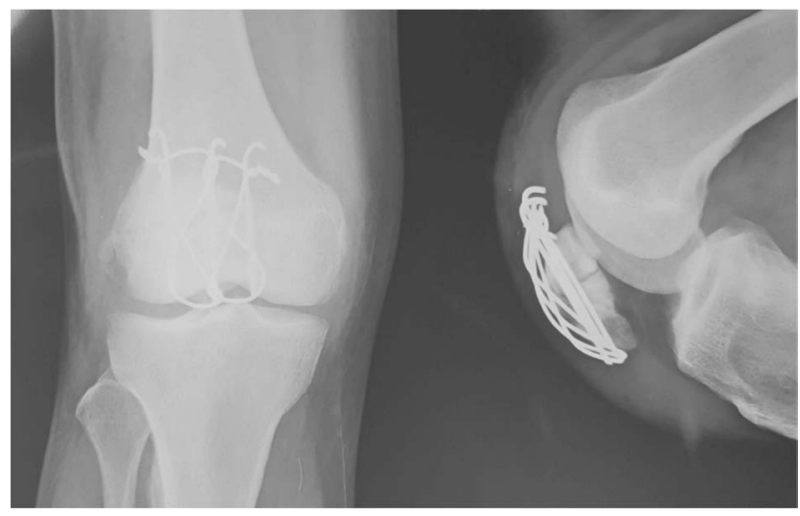

(c)

Figure 4. (a) Showing healed scar; (b) Showing good range of movement at 4 months follow up; (c) Showing the X-ray taken in maximum flexion in a well healed fracture.

and functional capability of the knee. The fracture union was achieved at an average of 10 weeks. The X-ray taken at 6 months, in maximal flexion shows a well healed fracture with implants in position (Figure 4(c)).

\section{Discussion}

The goals of treatment in the patellar fractures should aim for anatomic reduction, stable fixation with restoration of both, the articular surface and the extensor mechanism as well as the ability of early rehabilitation [2] [4] [5].

The study of the morphology of the patella and the patellar tendon revealed it to be significantly larger in males [6]. The males have larger patellae and no statistically significant difference between sides (i.e. right vs left patellae) [7].

The extent of the injury goes beyond the broken bone and often extends to the adjoining retinaculum. The transverse fractures of patella on which the TBW principle is used, after fixation are subject to varied forces that act at the fracture site [8]. It could be either in distraction due to the action of the quadriceps in extension or three-point bending that takes place on flexion of the knee. The premise of the tension band principles is successful only if three-point bending is achieved by flexing the knee. The technique described reinforces the fixation achieved and help to grab a larger area of the patella which often needs attention.

Though the technique interfragmentary screw fixation with the tension band principle seems to provide improved stability over the modified tension band or screws alone for transverse patella fractures has been described, it should be borne in mind that the technique is demanding [3].

When the modified tension band wiring technique is used and the wires are tightened, there is an opening of the patellar fragments from either the medial or lateral side depending upon the position of the $\mathrm{K}$ wires placed as well as the morphology of the patella. Concern about the fragment displacement of $2 \mathrm{~mm}$ was observed in $22 \%-30 \%$ of the patients [3].

The use of the additional third wire aims to neutralize the forces which can be beneficial for the fracture healing. The passage of an additional loop of the cer- 
clage wire over this $\mathrm{K}$ wire further contains the fracture fragments and provides stability till the eventual union.

\section{Conclusions}

We feel that this technique can be easily performed in cases where the occult fractures cannot be visualised on the X-rays or for those fracture lines which propagate during handling in the traditional TBW fixation.

This is a simple technique which allows the surgeon to extend the procedure by a few minutes, by passing an additional $\mathrm{K}$ wire and a second cerclage wire thereby adding more strength to the construct. Even though we could not do the biomechanical testing of the construct, we firmly believe that the technique is simple and can be easily carried out by anyone trained in musculoskeletal trauma surgery.

\section{Conflicts of Interest}

The authors declare no conflicts of interest regarding the publication of this paper.

\section{References}

[1] Ong, T.K., Chee, E.K., Wong, C.L. and Thevarajan, K. (2008) Fixation of Comminuted Patellar Fracture with Combined Cerclage and Tension Band Wiring Technique. Malaysian Orthopaedic Journal, 2, 40-42. https://doi.org/10.5704/MOJ.0811.010

[2] Gwinner, C., Märdian, S., Schwabe, P., Schaser, K.-D., Krapohl, B.D. and Jung, T.M. (2016) Current Concepts Review: Fractures of the Patella. GMS Interdisciplinary Plastic and Reconstructive Surgery DGPW, 5, Doc01.

[3] Gosal, H.S., Singh, P. and Field, R.E. (2001) Clinical Experience of Patellar Fracture Fixation Using Metal Wire or Non-Absorbable Polyester: A Study of 37 Cases. Injury, 32, 129-135. https://doi.org/10.1016/S0020-1383(00)00170-4

[4] Scilaris, T.A., Grantham, J.L., Prayson, M.J., et al. (1998) Biomechanical Comparison of Fixation Methods in Transverse Patella Fractures. Journal of Orthopaedic Trauma, 12, 356e359. https://doi.org/10.1097/00005131-199806000-00011

[5] Egol, K., Howard, D., Monroy, A., et al. (2014) Patella Fracture Fixation with Suture and Wire: You Reap What You Sew. Iowa Orthopedic Journal, 34, 63e67.

[6] Yoo, J.H., Yi, S.R. and Kim, J.H. (2007) The Geometry of Patella and Patellar Tendon Measured on Knee MRI. Surgical and Radiologic Anatomy, 29, 623-628. https://doi.org/10.1007/s00276-007-0261-x

[7] Shang, P., Zhang, L.N., Hou, Z.T., Bai, X.L., Ye, X., Xu, Z.B. and Huang, X. (2014) Morphometric Measurement of the Patella on 3D Model Reconstructed from CT Scan Images for the Southern Chinese Population. Chinese Medical Journal, 127, 96-101. https://doi.org/10.1109/ICIST.2014.6920508

[8] Lotke, A. and Ecker, M.L. (1981) Transverse Fractures of the Patella. Clinical Orthopaedics and Related Research, 158, 180-184.

https://doi.org/10.1097/00003086-198107000-00026 